\title{
THE RIEMANN PROBLEM FOR APPELL'S $\boldsymbol{F}_{4}$
}

\author{
Mitsuo Kato \\ (Received July 24, 1992) \\ (Revised Oct. 18, 1992)
}

Introduction

Let $z$ be an unknown and $r=z_{x x}, s=z_{x y}, t=z_{y y}, p=z_{x}, q=z_{y}$. The following system of differential equations is called Appell's system of type $F_{4}$ :

$\left(F_{4}\right) \quad\left\{\begin{array}{l}x(1-x) r-y^{2} t-2 x y s+\{\gamma-(\alpha+\beta+1) x\} p-(\alpha+\beta+1) y q-\alpha \beta z=0 \\ y(1-y) t-x^{2} r-2 x y s+\left\{\gamma^{\prime}-(\alpha+\beta+1) y\right\} q-(\alpha+\beta+1) x p-\alpha \beta z=0\end{array}\right.$

This system has singularities along $S:=\{x=0\} \cup\{y=0\} \cup\left\{(x-y)^{2}-2(x+y)+1=0\right\}$. Outside the singular locus $S$, $\left(F_{4}\right)$ has four linearly independent holomorphic solutions.

Assume that $\gamma, \gamma^{\prime} \neq 0,-1,-2, \ldots$ Then the Appell's hypergeometric series

$$
F_{4}\left(\alpha, \beta, \gamma, \gamma^{\prime} ; x, y\right)=\sum \frac{(\alpha, m+n)(\beta, m+n)}{(\gamma, m)\left(\gamma^{\prime}, n\right)(1, m)(1, n)} x^{m} y^{n}
$$

is a solution of $\left(F_{4}\right)$, where $(a, k)=a(a+1) \cdots(a+k-1)$.

Let $\mathscr{F}_{4}$ be the solution space of $\left(F_{4}\right)$, that is, the set of holomorphic functions on the universal covering space of $\mathbf{C}^{2}-S$ satisfing $\left(F_{4}\right)$.

In the natural way, the system of the differential equations $\left(F_{4}\right)$ can be extended to $\mathbf{P}^{2}$ or $\mathbf{P}^{1} \times \mathbf{P}^{1}$. The extended system has singularities also along the line at infinity $L_{\infty}$, or $\{x=\infty\} \cup\{y=\infty\}$.

In this paper we prove that $\mathscr{F}_{4}$ is characterized by the "exponents" at each irreducible component of the singular locus in the following way.

For generic $\alpha, \beta, \gamma, \gamma^{\prime}$,

$$
\mathscr{F}_{4}=P\left\{\begin{array}{cccc}
x=0 & y=0 & (x-y)^{2}-2(x+y)+1=0 & L_{\infty} \\
0 & 0 & 0 & \alpha \\
0 & 0 & 0 & \alpha \\
1-\gamma & 1-\gamma^{\prime} & 0 & \beta \\
1-\gamma & 1-\gamma^{\prime} & \gamma+\gamma^{\prime}-\alpha-\beta-\frac{1}{2} & \beta
\end{array}\right\}
$$


on $\mathbf{P}^{2}$ and

$$
\mathscr{F}_{4}=P\left\{\begin{array}{ccccc}
x=0 & y=0 & (x-y)^{2}-2(x+y)+1=0 & x=\infty & y=\infty \\
0 & 0 & 0 & \alpha & \alpha \\
0 & 0 & 0 & \beta & \beta \\
1-\gamma & 1-\gamma^{\prime} & 0 & 1+\alpha-\gamma^{\prime} & 1+\alpha-\gamma \\
1-\gamma & 1-\gamma^{\prime} & \gamma+\gamma^{\prime}-\alpha-\beta-\frac{1}{2} & 1+\beta-\gamma^{\prime} & 1+\beta-\gamma
\end{array}\right\}
$$

on $\mathbf{P}^{1} \times \mathbf{P}^{1}$.

For the meaning of "exponents", see the section 2 and for the meaning of the "P-function", see the section 3 .

The proof is divided into two steps. First (in the section 2) we determine the "exponents" of $\mathscr{F}_{4}$ at each irreducible component of the singular locus of $\left(F_{4}\right)$. Next (in the section 3 ) we show that any 4 dimensional vector space $\mathscr{F}$ consisting of holomorphic functions on the universal covering space of $\mathrm{C}^{2}-S$ must be $\mathscr{F}_{4}$ if $\mathscr{F}$ has the same "exponents" as $\mathscr{F}_{4}$.

For Appell's system $\left(F_{1}\right),\left(F_{2}\right),\left(F_{3}\right)$, the corresponding characterizations are known in Appell et Kampé de Fériet [1] and in Terada [3]. But $\left(F_{4}\right)$ has rather complicated singularities and has no Pfaffian system only with logarithmic singularities equivalent to itself. So it has been difficult to determine the "exponents" at the singularities.

The author thanks to professor Masaaki Yoshida and professor Toshiaki Terada for valuable advices.

\section{Existence of the solutions of a Pfaffian system}

Let $\Omega(x, y)=\Omega_{1} d y+\Omega_{2} d x$ be a holomorphic $(n, n)$ matrix with $d \Omega=\Omega \wedge \Omega$. Then it is well known that $d u=\Omega u$ has $n$-dimensional solution space. For later use we prove this existence theorem for $\Omega(x, y)$ with a logarithmic pole on $\{x=0\}$.

1.1 Theorem Let $A(y), B(x, y), \Omega_{2}(x, y)$ be $(n, n)$ matrices holomorphic on the closure of $D=\left\{(x, y)|| x|<r| y-,y_{0} \mid<s\right\}$. Put $\Omega_{1}=\frac{A}{x}+B, \Omega=\Omega_{1} d x$ $+\Omega_{2} d y$. Assume that $d \Omega=\Omega \wedge \Omega$ then $A(y)$ is conjugate to a constant matrix. 
If moreover none of the eigen values of $A(y)$ is a positive integer then $d u=\Omega u$ has $m$ linearly independent holomorphic solutions on $D$, where $m$ is the corank of $A(y)$.

\section{Proof.}

Put $Q(y)=\Omega_{2}(0, y)$. Then $d \Omega=\Omega \wedge \Omega$ restricted to $\{x=0\}$ implies $A^{\prime}(y)$ $=[Q, A]$. Let $P(y)$ be a fundamental solution of $P^{\prime}=Q P$. Then $P^{-1} A P$ is a constant matrix. If we replace $u$ by $P v$ then we obtain $d v=\widetilde{\Omega} v$ where $\tilde{\Omega}=\left(\frac{P^{-1} A P}{x}+\tilde{B}\right) d x+\tilde{\Omega}_{2} d y$. Hence $A$ is conjugate to a constant matrix. And we may assume that $A$ is a constant matrix with $[A, Q]=0$.

Let $u$ be holomorphic in $D$. Then $d u=\Omega u$ is equivalent to (1.1.A) $u_{x}=\Omega_{1} u, u_{y}=\Omega_{2} u$ which is again equivalent to (1.1.B 1) $u_{x}=\Omega_{1} u$, (1.1.B2) $u_{y}-\left.\Omega_{2} u\right|_{x=0}=0$.

We will prove this equivalence.

Let $u=\sum u_{k}(y) x^{k}, B=\sum B_{k}(y) x^{k}$. Then (1.1.B1), (1.1.B2) are equivalent to the following

(1.1.C1) $A u_{0}=0,(k-A) u_{k}=\sum_{i=0}^{k-1} B_{k-1-i} u_{i}$

(1.1.C2) $u_{0}^{\prime}=Q(y) u_{0}\left(Q(y)=\Omega_{2}(0, y)\right)$.

First note that the uniqueness property of (1.1.B1) holds. This means that if the "initial value" $u_{0}(y)=u(0, y)$ of a solution $u(x, y)$ of (1.1.B1) is zero then from (1.1.C1) all $u_{k}(y)=0$ hence $u=0$.

Assume (1.1.B1) and (1.1.B2). Put $v=u_{y}-\Omega_{2} u$. Then $\left.v\right|_{x=0}=0$ and

$$
\begin{aligned}
v_{x} & =u_{x y}-\Omega_{2} u_{x}-\Omega_{2 x} u=\left(\Omega_{1} u\right)_{y}-\Omega_{2} \Omega_{1} u-\Omega_{2 x} u \\
& =\Omega_{1}\left(u_{y}-\Omega_{2} u\right)+\left(\left[\Omega_{1}, \Omega_{2}\right]+\Omega_{1 y}-\Omega_{2 x}\right) u \\
& =\Omega_{1} v \quad(\text { by } d \Omega=\Omega \wedge \Omega) .
\end{aligned}
$$

This proves that $v=0$ that is (1.1.A).

Now we solve (1.1.C1), (1.1.C2) formally.

Since $A$ is commutable with $Q, A u_{0}=0$ and $u_{0}^{\prime}=Q u_{0}$ has $m$ linearly independent solutions. (1.1.C1) determines $u_{k}$ for each $u_{0}$.

We next show the convergence of the formal solutions. For any $u_{0}$, let $u_{k}$ be the solution of (1.1.C1) above. By the Cauchy's inequality we have 
$\left\|B_{k}\right\|<L_{1} / r^{k}(k=0,1,2, \ldots)$ for some positive number $L_{1}$. For some positive number $L_{2},\left\|(k-A)^{-1}\right\|<L_{2} / k$ holds for all $k(k=1,2,3 \ldots)$. Put $M=\left\|u_{0}\right\|$ and $L=L_{1} L_{2}$. Then using the second equality of (1.1.C1), we obtain the inequality

$$
\left\|u_{k}\right\|<\frac{M}{r^{k}}(1+r L)\left(1+\frac{r L}{2}\right) \cdots\left(1+\frac{r L}{k-1}\right) \frac{r L}{k}
$$

by the induction on $k$. Hence we have $\left\|u_{k}\right\|=o\left(r_{1}^{-k}\right)$ for any $r_{1}<r$. This proves the convergence of $u$.

This completes the proof of the theorem 1.1.

\section{The exponents of $\widetilde{\mathscr{F}_{4}}$ along the singular sets}

\subsection{Exponent of $\mathscr{\mathscr { F }}_{4}$ along $\{x=0\}$.}

Assume that $\gamma$ is not an integer.

Let $\phi: \mathbf{C}^{2} \rightarrow \mathbf{C}^{2}$ be a 2 -sheeted covering defined by $\phi(X, Y)=(X Y,(1-X)$ $(1-Y)) . \phi$ is locally biholomorphic at $X=0, Y=Y_{0} \neq 0$.

As is stated in Kato [2], the pull back $\phi^{*}\left(F_{4}\right)$ of $\left(F_{4}\right)$ is equivalent to a Pfaffian system $d v=P v$, where $v={ }^{t}\left(z, X z_{X}, Y z_{Y}, X Y\left(z_{X Y}+\varepsilon\left(z_{X}-z_{Y}\right) /(X-Y)\right)\right)$, $\varepsilon=\gamma+\gamma^{\prime}-\alpha-\beta-1$ and $P=P_{1} \frac{d X}{X}+P_{2} \frac{d Y}{Y}+P_{3} \frac{d X}{X-1}+P_{4} \frac{d Y}{Y-1}+$ $P_{5} \frac{d(X-Y)}{X-Y}$ with constant $(4,4)$ matrices

$$
\begin{aligned}
& P_{1}=\left(\begin{array}{cccc}
0 & 1 & 0 & 0 \\
0 & 1-\gamma & 0 & 0 \\
0 & \varepsilon & 0 & 1 \\
0 & 0 & 0 & 1-\gamma
\end{array}\right), \quad P_{2}=\left(\begin{array}{cccc}
0 & 0 & 1 & 0 \\
0 & 0 & \varepsilon & 1 \\
0 & 0 & 1-\gamma & 0 \\
0 & 0 & 0 & 1-\gamma
\end{array}\right), \\
& P_{3}=\left(\begin{array}{cccc}
0 & 0 & 0 & 0 \\
-\alpha \beta & -\gamma^{\prime} & \varepsilon & 0 \\
0 & 0 & 0 & 0 \\
0 & 0 & -(\alpha+\varepsilon)(\beta+\varepsilon) & -\gamma^{\prime}
\end{array}\right),
\end{aligned}
$$




$$
\begin{aligned}
& P_{4}=\left(\begin{array}{cccc}
0 & 0 & 0 & 0 \\
0 & 0 & 0 & 0 \\
-\alpha \beta & \varepsilon & -\gamma^{\prime} & 0 \\
0 & -(\alpha+\varepsilon)(\beta+\varepsilon) & 0 & -\gamma^{\prime}
\end{array}\right), \\
& P_{5}=\left(\begin{array}{cccc}
0 & 0 & 0 & 0 \\
0 & \varepsilon & -\varepsilon & 0 \\
0 & -\varepsilon & \varepsilon & 0 \\
0 & 0 & 0 & 0
\end{array}\right) .
\end{aligned}
$$

Put $P=\left(\frac{A}{X}+B\right) d X+C d Y$ and $Q=C(0, Y)$ then $A=P_{1}$ and $Q=\left(P_{2}+P_{5}\right)$ $\frac{1}{Y}+P_{4} \frac{1}{Y-1}$.

The solution $v_{0}$ of $A v_{0}=0$ and $v_{0}^{\prime}=Q v_{0}$ consists of ${ }^{t}\left(z(0,1-Y), 0,-Y z_{Y}\right.$ $(0,1-Y), 0)$ with $z(0, y)$ being a solution of $\left(F\left(\alpha, \beta, \gamma^{\prime} ; y\right)\right)$.

This means that for any solution $z_{0}(y)$ of $\left(F\left(\alpha, \beta, \gamma^{\prime} ; y\right)\right)$, there exists a unique holomorphic solution $z(x, y)$ of $\left(F_{4}\right)$ in a neighborhood of $\left(0, y_{0}\right)$ with $z(0, y)=z_{0}(y)$.

Next we know that $z=x^{1-\gamma} f(x, y)$ is a solution of $\left(F_{4}\left(\alpha, \beta, \gamma, \gamma^{\prime}\right)\right)$ if and only if $f(x, y)$ is a solution of $\left(F_{4}\left(\alpha-\gamma+1, \beta-\gamma+1,2-\gamma, \gamma^{\prime}\right)\right)$ (Appell et Kampé de Fériet [1]).

Thus we have proved that if $\gamma$ is not an integer then $\mathscr{F}_{4}$ has the exponent $(0,0,1-\gamma, 1-\gamma)$ along $\{x=0\}$. This means, by definition, that at any point $\left(0, y_{0}\right) \quad\left(y_{0} \neq 0,1, \infty\right)$, there exist holomorphic functions $g_{1}(x, y), g_{2}(x, y)$, $g_{3}(x, y), g_{4}(x, y)$ so that $g_{1}, g_{2}, x^{1-\gamma} g_{3}, x^{1-\gamma} g_{4}$ form a basis of $\mathscr{F}_{4}$ and that both pairs $g_{1}(0, y), g_{2}(0, y)$ and $g_{3}(0, y), g_{4}(0, y)$ are linearly independent respectively.

In fact $\mathscr{F}_{4}$ has the following four special elements provided that neither of $\gamma, \gamma^{\prime}$ is an integer

(Appell et Kampé de Fériet [1]):

$F_{4}\left(\alpha, \beta, \gamma, \gamma^{\prime} ; x, y\right)$

$x^{1-\gamma} F_{4}\left(1+\alpha-\gamma, 1+\beta-\gamma, 2-\gamma, \gamma^{\prime} ; x, y\right)$,

$y^{1-\gamma^{\prime}} F_{4}\left(1+\alpha-\gamma^{\prime}, 1+\beta-\gamma^{\prime}, \gamma, 2-\gamma^{\prime} ; x, y\right)$,

$x^{1-\gamma} y^{1-\gamma^{\prime}} F_{4}\left(2+\alpha-\gamma-\gamma^{\prime}, 2+\beta-\gamma-\gamma^{\prime}, 2-\gamma, 2-\gamma^{\prime} ; x, y\right)$. 
2.2 Exponent along $\{\boldsymbol{y}=\mathbf{0}\}$.

Assume that $\gamma^{\prime}$ is not an integer, then $\mathscr{F}_{4}$ has the exponent $\left(0,0,1-\gamma^{\prime}, 1-\gamma^{\prime}\right)$ along $\{y=0\}$.

\subsection{Exponent along $\boldsymbol{L}_{\infty}$.}

Assume that $\alpha-\beta$ is not an integer.

The direct calculations show that $z=y^{-\alpha} w(x / y, 1 / y)$ is a solution of $\left(F_{4}\right)$ if and only if $w(\xi, \eta)$ is a solution of $\left(F_{4}\left(\alpha, \alpha-\gamma^{\prime}+1, \gamma, \alpha-\beta+1 ; \xi, \eta\right)\right)$. So from 2.2, $\mathscr{F}_{4}$ has the exponent $(\alpha, \alpha, \beta, \beta)$ along $L_{\infty}$.

\subsection{Exponent along $\left\{(x-y)^{2}-2(x+y)+1=0\right\}$.}

Assume that $\gamma+\gamma^{\prime}-\alpha-\beta-\frac{1}{2}$ is not an integer. Put $D=(x-y)^{2}$ $-2(x+y)+1$.

As in the section 2.1, we put $\varepsilon=\gamma+\gamma^{\prime}-\alpha-\beta-1$ and consider the $2: 1$ mapping $\phi(X, Y)=(X Y,(1-X)(1-Y))$. Then as is stated in 2.1, the pull back $\phi^{*}\left(F_{4}\right)$ of $\left(F_{4}\right)$ is equivalent to

$$
d v=P v .
$$

The pull back of $\{D=0\}$ is $\{X-Y=0\}$.

$$
\text { Put } \xi=\frac{X-Y}{2}, \eta=\frac{X+Y}{2} \text { and } u=\left(\begin{array}{cccc}
1 & 0 & 0 & 0 \\
0 & 1 & -1 & 0 \\
0 & 1 & 1 & 0 \\
0 & 0 & 0 & 1
\end{array}\right) v
$$

Then (2.4.A) takes the form

$$
\begin{aligned}
d u & =\Omega u \\
& =\left\{\left(\frac{1}{\xi} A+B\right) d \xi+\Omega_{2} d \eta\right\} u .
\end{aligned}
$$

Where

$$
A=\left(\begin{array}{cccc}
0 & 0 & 0 & 0 \\
0 & 2 \varepsilon & 0 & 0 \\
0 & 0 & 0 & 0 \\
0 & 0 & 0 & 0
\end{array}\right)
$$


$B=q(\xi, \eta)\left(Q_{1}-\frac{\xi}{\eta} Q_{2}\right)+q(\xi, \eta-1)\left(Q_{3}-\frac{\xi}{\eta-1} Q_{4}\right)$

$\Omega_{2}=q(\xi, \eta)\left(Q_{2}-\frac{\xi}{\eta} Q_{1}\right)+q(\xi, \eta-1)\left(Q_{4}-\frac{\xi}{\eta-1} Q_{3}\right)$,

$q(\xi, \eta)=\frac{\eta}{\eta^{2}-\xi^{2}}$

$Q_{1}, Q_{3}$ are constant matrices of the form $\left(\begin{array}{llll}0 & * & 0 & 0 \\ * & 0 & * & * \\ 0 & * & 0 & 0 \\ 0 & * & 0 & 0\end{array}\right)$,

$Q_{2}, Q_{4}$ are constant matrices of the form $\left(\begin{array}{cccc}* & 0 & * & * \\ 0 & * & 0 & 0 \\ * & 0 & * & * \\ * & 0 & * & *\end{array}\right)$

More presicely $Q_{2}$ is of the form $\left(\begin{array}{llll}0 & 0 & 1 & 0 \\ 0 & * & 0 & 0 \\ 0 & 0 & * & 2 \\ 0 & 0 & 0 & *\end{array}\right)$

$Q_{4}$ is of the form

$$
\left(\begin{array}{cccc}
0 & 0 & 0 & 0 \\
0 & * & 0 & 0 \\
* & 0 & * & 0 \\
0 & 0 & * & *
\end{array}\right)
$$

Put $Q(\eta)=\Omega_{2}(0, \eta)=\frac{1}{\eta} Q_{2}+\frac{1}{\eta-1} Q_{4}$

For $u=\xi^{\lambda} w, w=w_{0}(\eta)+\xi w_{1}(\eta)+\xi^{2} w_{2}(\eta)+\cdots,(2.4 . \mathrm{B})$ is equivalent to the following (2.4.C1) and (2.4.C2).

$$
w_{\xi}=\left(\frac{1}{\xi}(A-\lambda E)+B\right) w
$$




$$
w_{\eta}=Q w
$$

which implies the following $\left(2.4 \cdot \mathrm{EQ}_{k}\right)(k=0,1,2, \ldots)$ and $\left(2.4 . \mathrm{EQ}_{\eta}\right)$.

$$
\begin{aligned}
& (\lambda+k-A) w_{k}= \\
& \quad \frac{1}{\eta} Q_{1}\left(w_{k-1}+\frac{w_{k-3}}{\eta^{2}}+\frac{w_{k-5}}{\eta^{4}}+\cdots\right) \\
& +\frac{1}{\eta-1} Q_{3}\left(w_{k-1}+\frac{w_{k-3}}{(\eta-1)^{2}}+\frac{w_{k-5}}{(\eta-1)^{4}}+\cdots\right) \\
& -\frac{1}{\eta^{2}} Q_{2}\left(w_{k-2}+\frac{w_{k-4}}{\eta^{2}}+\cdots\right)-\frac{1}{(\eta-1)^{2}} Q_{4}\left(w_{k-2}+\frac{w_{k-4}}{(\eta-1)^{2}}+\cdots\right)
\end{aligned}
$$

$$
w_{0}(\eta)^{\prime}=Q w_{0} .
$$

Since $A-\lambda$ may have a positive integer as its eigen value, $\left(2.4 . \mathrm{EQ}_{k}\right)$ and (2.4.EQ ${ }_{\eta}$ ) does not imply (2.4.B) (but see the Lemma 2.4 .2 below).

Corresponding to the eigen values 0 and $2 \varepsilon$ of $A,\left(2.4 . \mathrm{EQ}_{\eta}\right)$ has two invariant vector spaces $V_{0}$ and $V_{1}: V_{0}=\left\{t\left(f_{0}(\eta), 0, g_{0}(\eta), h_{0}(\eta)\right)\right\}, \quad V_{1}=\left\{t\left(0, f_{1}(\eta)\right.\right.$, $0,0)\}$, where $f_{0}, g_{0}, h_{0}, f_{1}$ are functions of $\eta$.

For $k=0$ and 1 we have

$$
\text { (2.4.QV) } \quad Q_{i} V_{k} \subset V_{k} \text { for } i=2,4 \text { and } Q_{i} V_{k} \subset V_{1-k} \text { for } i=1,3 \text {. }
$$

\subsection{1}

We assume $\lambda=0$ or $2 \varepsilon$. It is clear that

if $\varepsilon \neq 0$ and $\lambda=0$ then $\left(2.4 . \mathrm{EQ}_{0}\right)$ implies $w_{0} \in V_{0}$,

if $\varepsilon \neq 0$ and $\lambda=2 \varepsilon$ then (2.4.EQ $\mathrm{EQ}_{0}$ ) implies $w_{0} \in V_{1}$,

if $\varepsilon=0$ and $\lambda=0$ then $\left(2.4 . \mathrm{EQ}_{0}\right)$ holds for any $w_{0}$.

Lemma Assume $\varepsilon+\frac{1}{2}$ is not an integer.

Let $\lambda=0$, then for any $w_{0} \in V_{0}$ there exists a unique solution $w_{k}$ of $\left(2.4 . \mathrm{EQ}_{k}\right)$ $k=1,2,3, \ldots$ satisfing the following property:

$$
w_{2 k} \in V_{0} \quad \text { and } \quad w_{2 k+1} \in V_{1} \quad k \geq 0 .
$$

Let $\lambda=2 \varepsilon$, then for any $w_{0} \in V_{1}$ there exists a unique solution $w_{k}$ of (2.4.EQ $\mathrm{E}_{k}$ ) satisfing the following property:

$$
w_{2 k} \in V_{1} \quad \text { and } \quad w_{2 k+1} \in V_{0} \quad k \geq 0 .
$$


Proof This follows from 2.4.1.1 2.4.1.3 below.

2.4.1.1 Except for the case $\lambda=0$ and $2 \varepsilon$ is a positive integer or the case $\lambda=2 \varepsilon$ and $2 \varepsilon$ is a negative integer, for any $w_{0},\left(2.4 . \mathrm{EQ}_{k}\right)$ has the unique solution $w_{k}$. If $w_{0} \in V_{0}$ then $w_{k}$ satisfies (2.4.1.V0). If $w_{0} \in V_{1}$ then $w_{k}$ satisfies (2.4.1.V1).

Proof $(\lambda+n) E-A$ is singular only in the exceptional case stated above. From (2.4.QV), (2.4.1.V0) or (2.4.1.V1) holds inductively.

2.4.1.2 Assume $\lambda=0$ and $\varepsilon$ is a positive integer. Then for any $w_{0} \in V_{0}$, $\left(2.4 . \mathrm{EQ}_{k}\right)$ has the unique solution $w_{k}$ if we require the property $(2.4 .1 . \mathrm{V} 0)$.

Proof Put $\varepsilon=m$. Then $\left(2.4 . \mathrm{EQ}_{k}\right)$ has the unique solution $w_{k}$ up to $k=2 m-1$ with the property (2.4.1.V0). Hence the right hand side of $\left(2.4 . \mathrm{EQ}_{2 m}\right)$ belongs to $V_{0}$ by $(2.4 . \mathrm{QV})$, and the left hand side is $(2 m E-A) w_{2 m}$. Thus (2.4.EQ $\left.\mathrm{EQ}_{2 m}\right)$ has the unique solution $w_{2 m}$ if we restrict $w_{2 m}$ to $V_{0}\left(w_{2 m}+V_{1}\right.$ is the general solution). For $k>2 m\left(2.4 . \mathrm{EQ}_{k}\right)$ has the unique solution $w_{k}$ with the property (2.4.1.V0). This completes the proof.

2.4.1.3 Assume $\lambda=2 \varepsilon$ and $\varepsilon$ is a negative integer. Then for any $w_{0} \in V_{1}$, $\left(2.4 . \mathrm{EQ}_{k}\right.$ ) has the unique solution $w_{k}$ if we require the property (2.4.1.V1).

Proof Let $\lambda=2 \varepsilon=-2 m$ for some positive integer $m$. Then (2.4.EQ $k$ ) has the unique solution $w_{k}$ up to $k=2 m-1$ with the property (2.4.1.V1). Hence the right hand side of $\left(2.4 . \mathrm{EQ}_{2 m}\right)$ belongs to $V_{1}$ by $(2.4 . \mathrm{QV})$, and the left hand side is $-A w_{2 m}$. Thus $\left(2.4 . \mathrm{EQ}_{2 m}\right)$ has the unique solution $w_{2 m}$ if we restrict $w_{2 m}$ to $V_{1}\left(w_{2 m}+V_{0}\right.$ is the general solution). For $k>2 m\left(2.4 . \mathrm{EQ}_{k}\right)$ has the unique solution $w_{k}$ with the property (2.4.1.V1). This completes the proof.

\subsubsection{Lemma}

For $u=\xi^{\lambda} w, w=w_{0}(\eta)+\xi w_{1}(\eta)+\xi^{2} w_{2}(\eta)+\cdots,\left(2.4 . \mathrm{EQ}_{k}\right)$ and $\left(2.4 . \mathrm{EQ}_{\eta}\right)$ are sufficient conditions for (2.4.B) in the following sence.

In the case when $\lambda=0$. If $w_{0}(\eta) \in V_{0}$ satisfies $\left(2.4 . \mathrm{EQ}_{\eta}\right)$ and $w_{k}$ satisfies (2.4.EQ $\mathrm{E}_{k}$ ) together with the property (2.4.1.V0), then $u=w$ satisfies (2.4.B).

In the case when $\lambda=2 \varepsilon$. If $w_{0}(\eta) \in V_{1}$ satisfies $\left(2.4 . \mathrm{EQ}_{\eta}\right)$ and $w_{k}$ satisfies (2.4.EQ $\left.\mathrm{E}_{k}\right)$ together with the property (2.4.1.V1), then $u=\xi^{\lambda} w$ satisfies (2.4.B).

Proof Recall that $Q=\left(\frac{1}{\xi} A+B\right) d \xi+\Omega_{2} d \eta$ and that (2.4.B) is equivalent 
to (2.4.C1) and (2.4.C2). Put $\psi=w_{\eta}-\Omega_{2} w$. Then we have to show that $\left.\psi\right|_{\xi=0}=0$ implies $\psi=0$.

By the integrabity condition $d \Omega=\Omega \wedge \Omega$, $\psi$ also satisfies (2.4.C1).

If $\lambda=0$ then $w_{k}$ satisfies (2.4.1.V0) so $\psi$ also satisfies (2.4.1.V0).

If $\lambda=2 \varepsilon$ then $w_{k}$ satisfies (2.4.1.V1) so $\psi$ also satisfies (2.4.1.V1).

Hence we can apply the uniqueness property in the lemma 2.4.1 to $\psi$. Since $\left.\psi\right|_{\xi}=0$, we have $\psi=0$. This completes the proof.

\subsubsection{The formal existence of the solution of (2.4.B)}

We first construct the formal solutions of (2.4.B) of the form $u=\xi^{\lambda} w$, $w=w_{0}(\eta)+\xi w_{1}(\eta)+\xi^{2} w_{2}(\eta)+\cdots, w_{0} \neq 0, \lambda=0$ or $2 \varepsilon$.

Let $\lambda=0$. Since $V_{0}$ is an invariant subspace of $\left(2.4 . \mathrm{EQ}_{\eta}\right),\left(2.4 . \mathrm{EQ}_{\eta}\right)$ has a 3 dimensional solution space in $V_{0}$. Let $w_{0}=(f, 0, g, h) \in V_{0}$ be any nonzero solution of $\left(2.4 . \mathrm{EQ}_{\eta}\right)$. If $f=0$ then $\left(2.4 . \mathrm{EQ}_{\eta}\right)$ implies that $g=h=0$. That is, $w_{0} \neq 0$ means $f \neq 0$. By the lemma 2.4.1, there exists $w=w_{0}+\xi w_{1}+\cdots$ satisfing (2.4.EQ $\mathrm{EQ}_{k}$ ) and (2.4.1.V0). Again by 2.4.2 $u=w$ is a solution of (2.4.B).

Let $\lambda=2 \varepsilon . \quad V_{1}$ is also an invariant subspace of $\left(2.4 . \mathrm{EQ}_{\eta}\right)$ The solution $w_{0} \in V_{1}$ of $\left(2.4 . \mathrm{EQ}_{\eta}\right)$ is of the form $\left(0, c \eta^{\mu}(\eta-1)^{\nu}, 0,0\right)$. By 2.4.1, there exists $w=w_{0}+\xi w_{1}+\cdots$ satisfing $\left(2.4 . \mathrm{EQ}_{k}\right)$ and (2.4.1.V1). Then by 2.4.2, $u=\xi^{\lambda} w$ is a solution of (2.4.B). We note $w_{1}=\left(\frac{c}{2 \varepsilon+1} \eta^{\mu-1}(\eta-1)^{v}, 0, *, *\right)$ for later use.

\subsubsection{The Convergence of the power series $w$}

Assume that $w_{k}(\eta)$ are holomorphic functions satisfing (2.4.EQ $\mathrm{E}_{k}$ ). Then $w=w_{0}+\xi w_{1}+\xi^{2} w_{2}+\cdots$ converges if $|\xi|<|\eta|,|1-\eta|$.

Proof The proof is the same as that of Theorem 1.1. Note that $\xi= \pm \eta$, $\xi= \pm(1-\eta)$ means that $X Y=0,(1-X)(1-Y)=0$ respectively.

\subsubsection{The exponent of $\mathscr{F}_{4}$ along $\{D=0\}$}

Recall that $D=(x-y)^{2}-2(x+y)+1$.

We will construct solutions of $\left(F_{4}\right)$ from solutions of (2.4.B).

Let $\lambda=0$. Then (2.4.B) has three independent solutions $u_{1}, u_{2}, u_{3}$ with the property (2.4.1.V0). Put $u_{k}={ }^{t}\left(g_{k}, *, *, *\right)$. Then the property (2.4.1.V0) guarantees the fact that $g_{k}$ are even functions of $\xi$. The linealy independence of $u_{k} k=1,2,3$ implies that of $u_{k}(0, \eta) k=1,2,3$ and that of $g_{k}(0, \eta) k=1,2,3$ (cf 2.4.1, 2.4.3). Since $\xi^{2}=D / 4$ and $\eta=(1+x-y) / 2, g_{k}$ are holomorphic 
functions of $(x, y)$ which are also denoted by $g_{k}(x, y)$. Thus obtained $g_{k}(x, y)$ are solutions of $\left(F_{4}\right)$ and are linearly independent when they are restricted to $\{D=0\}$.

Let $\lambda=2 \varepsilon$. Then (2.4.B) has a solution $u=\xi^{\lambda} w$ with the property (2.4.1.V1). Put $u={ }^{t}(g, *, *, *)$. Then $g$ is of the form $\xi^{\lambda+1} g_{4}$ where $g_{4}$ is an even function of $\xi$ and $g_{4}(0, \eta)=\frac{c}{2 \varepsilon+1} \eta^{\mu-1}(\eta-1)^{v} \neq 0$ as stated in 2.4.3. Again $g_{4}$ may be considered a holomorphic function of $(x, y)$. $g=D^{\varepsilon+1 / 2} g_{4}(x, y)$ is a solution of $\left(F_{4}\right)$ and the restriction of $g_{4}$ to $\{D=0\}$ is not zero.

Thus we have proved the following result.

If $\gamma+\gamma^{\prime}-\alpha-\beta-\frac{1}{2}\left(=\varepsilon+\frac{1}{2}\right)$ is not an integer, then at each point $(a, b)$ with $D(a, b)=0, a b \neq 0, \quad\left(F_{4}\right)$ has solutions $g_{1}(x, y), g_{2}(x, y), g_{3}(x, y)$, $D^{\gamma+\gamma^{\prime}-\alpha-\beta-\frac{1}{2}} g_{4}(x, y)$ so that $g_{1}, g_{2}, g_{3}, g_{4}$ are holomorphic at $(a, b)$, and the restrictions of $g_{1}, g_{2}, g_{3}$ to $\{D=0\}$ are linearly independent, and the restriction of $g_{4}$ to $\{D=0\}$ is not (identically) zero. This means, by definition, that $\mathscr{F}_{4}$ has the exponent $\left(0,0,0, \gamma+\gamma^{\prime}-\alpha-\beta-\frac{1}{2}\right)$ along $\{D=0\}$.

\subsection{Exponent along $\{y=\infty\}$ in $\mathbf{P}^{1} \times \mathbf{P}^{1}$}

Assume that the difference of any two members of $\{\alpha, \beta, 1+\alpha-\gamma$, $1+\beta-\gamma\}$ is not an integer.

As stated in 2.3, $y^{-\alpha} w(x / y, 1 / y)$ belongs to $\mathscr{F}_{4}$ if $w(\xi, \eta)$ is a solution of $\left(F_{4}\left(\alpha, \alpha-\gamma^{\prime}+1, \gamma, \alpha-\beta+1 ; \xi, \eta\right)\right)$. In particular

$z=y^{-\alpha} F_{4}\left(\alpha, \alpha-\gamma^{\prime}+1, \gamma, \alpha-\beta+1 ; x / y, 1 / y\right)$,

$z=y^{-(1+\alpha-\gamma)} x^{1-\gamma} F_{4}\left(\alpha-\gamma+1, \alpha-\gamma-\gamma^{\prime}+2,2-\gamma, \alpha-\beta+1 ; x / y, 1 / y\right)$,

$z=y^{-\beta} F_{4}\left(\beta, \beta-\gamma^{\prime}+1, \gamma, \beta-\alpha+1 ; x / y, 1 / y\right)$,

$z=y^{-(1+\beta-\gamma)} x^{1-\gamma} F_{4}\left(\beta-\gamma+1, \beta-\gamma-\gamma^{\prime}+2,2-\gamma, \beta-\alpha+1 ; x / y, 1 / y\right)$

are solutions near $\{y=\infty\}$.

Thus $\mathscr{F}_{4}$ has the exponent $(\alpha, \beta, 1+\alpha-\gamma, 1+\beta-\gamma)$ at $\{y=\infty\}$. This means, by definition, $\mathscr{F}_{4}$ has a basis $y^{-\alpha} g_{1}, y^{-\beta} g_{2}, y^{-(1+\alpha-\gamma)} g_{3}, y^{-(1+\beta-\gamma)} g_{4}$, where $g_{k}$ are holomorphic at $(a, \infty)(a \neq 0, \infty)$ and the restrictions $g_{k}(x, \infty)$ are not zero. In our case, $g_{1}(x, \infty)=g_{2}(x, \infty)=1$ and $g_{3}(x, \infty)=g_{4}(x, \infty)=$ $x^{1-\gamma}$. 
2.6 Exponent of $\mathscr{\mathscr { F }}_{4}$ along $\{\boldsymbol{x}=\infty\}$

Assume that the difference of any two members of $\left\{\alpha, \beta, 1+\alpha-\gamma^{\prime}\right.$, $\left.1+\beta-\gamma^{\prime}\right\}$ is not an integer. Then $\mathscr{F}_{4}$ has the exponent $\left(\alpha, \beta, 1+\alpha-\gamma^{\prime}\right.$, $\left.1+\beta-\gamma^{\prime}\right)$ along $\{x=\infty\}$.

\section{Riemann's Problem of $\left(F_{4}\right)$ on $\mathrm{P}^{2}$}

We consider $(x, y)$ to be the inhomogeneous coordinate of $\mathbf{P}^{2}$. Let again $D=(x-y)^{2}-2(x+y)+1$. Let $L_{x}, L_{y}$ and $C$ be subsets of $\mathbf{P}^{2}$ defined by $x=0, y=0$ and $D=0$ respectively.

Assume that none of $1-\gamma, 1-\gamma^{\prime}, \alpha-\beta, \gamma+\gamma^{\prime}-\alpha-\beta-1 / 2$ is zero and put

$$
\mathscr{F}=P\left\{\begin{array}{cccc}
x=0 & y=0 & (x-y)^{2}-2(x+y)+1=0 & L_{\infty} \\
0 & 0 & 0 & \alpha \\
0 & 0 & 0 & \alpha \\
1-\gamma & 1-\gamma^{\prime} & 0 & \beta \\
1-\gamma & 1-\gamma^{\prime} & \gamma+\gamma^{\prime}-\alpha-\beta-\frac{1}{2} & \beta
\end{array}\right\} .
$$

This means that $\mathscr{F}$ is a 4 dimensional vector space consisting of holomorphic functions on the universal covering space of $\mathbf{P}^{2}-L_{x} \cup L_{y} \cup L_{\infty} \cup C$ with the following properties.

$\left(\operatorname{EXP}_{x}\right): \quad \mathscr{F}$ has the exponent $(0,0,1-\gamma, 1-\gamma)$ along $L_{x}$. This means, by definition, that at each point of $L_{x}-L_{y} \cup L_{\infty} \cup C$ there exist an open neighbourhood $U$ and holomorphic functions $g_{1}, g_{2}, g_{3}, g_{4}$ on $U$ so that $g_{1}, g_{2}, x^{1-\gamma} g_{3}, x^{1-\gamma} g_{4}$ form a basis of $\mathscr{F}$ and that both of the pairs $g_{1}, g_{2}$ and $g_{3}, g_{4}$ are linearly independent when they are restricted to $L_{x}$.

$\left(\mathrm{EXP}_{y}\right): \mathscr{F}$ has the exponent $\left(0,0,1-\gamma^{\prime}, 1-\gamma^{\prime}\right)$ along $L_{y}$.

$\left(\mathrm{EXP}_{\infty}\right)$ : $\mathscr{F}$ has the exponent $\{\alpha, \alpha, \beta, \beta)$ along $L_{\infty}$. This means, by definition, that at each point of $L_{\infty}-L_{x} \cup L_{y} \cup C$ there exist an open neighbourhood $U$ in $\mathbf{P}^{2}$ and holomorphic functions $g_{1}, g_{2}, g_{3}, g_{4}$ on $U$ and coordinate functions $\xi, \eta$ with $L_{\infty} \cap U=\{\xi=0\}$ so that $\xi^{\alpha} g_{1}, \xi^{\alpha} g_{2}, \xi^{\beta} g_{3}, \xi^{\beta} g_{4}$ form a basis of $\mathscr{F}$ and that both of the pairs $g_{1}, g_{2}$ and $g_{3}, g_{4}$ are linearly independent when they are restricted to $L_{\infty}$.

$\left(\operatorname{EXP}_{C}\right): \mathscr{F}$ has the exponent $\left(0,0,0, \gamma+\gamma^{\prime}-\alpha-\beta-\frac{1}{2}\right)$ along $C$. This 
means, by definition, that at each point of $C-L_{x} \cup L_{y} \cup L_{\infty}$ there exist an open neighbourhood $U$ and holomorphic functions $g_{1}, g_{2}, g_{3}, g_{4}$ on $U$ so that $g_{1}, g_{2}, g_{3}, D^{\gamma+\gamma^{\prime}-\alpha-\beta-1 / 2} g_{4}$ form a basis of $\mathscr{F}$ and that the restrictions of $g_{1}, g_{2}, g_{3}$ to $C$ are linearly independent and that of $g_{4}$ is not (identically) zero.

Under these conditions we shall prove that $\mathscr{F}$ is the solution space $\mathscr{F}_{4}$ of the Appell's differential equations $\left(F_{4}\right)$.

\section{1}

Fix a basis $f_{1}, f_{2}, f_{3}, f_{4}$ of $\mathscr{F}$.

Let $f$ be any element of $\mathscr{F}$. Then we shall prove that $f$ belongs to $\mathscr{F}_{4}$, that is, both of

$$
\begin{aligned}
& Q_{1}(f)=x(1-x) f_{x x}-y^{2} f_{y y}-2 x y f_{x y}+(\gamma-(\alpha+\beta+1) x) f_{x}-(\alpha+\beta+1) y f_{y}-\alpha \beta f \\
& Q_{2}(f)=y(1-y) f_{y y}-x^{2} f_{x x}-2 x y f_{x y}+\left(\gamma^{\prime}-(\alpha+\beta+1) y\right) f_{y}-(\alpha+\beta+1) x f_{x}-\alpha \beta f
\end{aligned}
$$

are zero.

We define the differential operator $D_{2}$ by $D_{2}(f)=x f_{x x}+y f_{y y}+(x+y-1) f_{x y}$. Expanding the following determinant (which is identically zero)

$$
\left|\begin{array}{lllll}
f_{1} & f_{2} & f_{3} & f_{4} & f \\
f_{1 x} & f_{2 x} & f_{3 x} & f_{4 x} & f_{x} \\
f_{1 y} & f_{2 y} & f_{3 y} & f_{4 y} & f_{y} \\
D_{2}\left(f_{1}\right) & D_{2}\left(f_{2}\right) & D_{2}\left(f_{3}\right) & D_{2}\left(f_{4}\right) & D_{2}(f) \\
Q_{i}\left(f_{1}\right) & Q_{i}\left(f_{2}\right) & Q_{i}\left(f_{3}\right) & Q_{i}\left(f_{4}\right) & Q_{i}(f)
\end{array}\right| x^{2 \gamma} y^{2 \gamma^{\prime}} D^{-\gamma-\gamma^{\prime}+\alpha+\beta+\frac{3}{2}}
$$

by the last column, we obtain the equation

$$
\Delta_{0}^{i} f-\Delta_{1}^{i} f_{x}+\Delta_{2}^{i} f_{y}-\Delta_{3}^{i} D_{2}(f)+\Delta Q_{i}(f)=0 \quad i=1,2 .
$$

We will prove that $\Delta$ is a nonzero constant and $\Delta_{k}^{i}(i=1,2 k=0,1,2,3)$ is identically zero.

\subsection{Local behavior of $\Delta, \Delta_{k}^{i}$}

We note that if we exchange the basis $f_{1}, f_{2}, f_{3}, f_{4}$ then $\Delta_{k}^{i}$ and $\Delta$ change to $c \Delta_{k}^{i}$ and $c \Delta$ for some nonzero constant $c$.

\subsubsection{Behavior along $L_{x}$}

For each point of $L_{x}-L_{y} \cup L_{\infty} \cup C$, take a neibourhood $U$ disjoint from $L_{y} \cup L_{\infty} \cup C$ and a basis $g_{1}, g_{2}, x^{1-\gamma} g_{3}, x^{1-\gamma} g_{4}$ of $\mathscr{F}$ satisfing $\left(\mathrm{EXP}_{x}\right)$. 
Then

$$
\begin{aligned}
& D_{2}\left(x^{1-\gamma} g_{k}\right)=x^{-\gamma}\left((1-\gamma)\left(-\gamma g_{k}+(y-1) g_{k y}\right)+x g_{k}^{\prime}\right) \\
& Q_{i}\left(x^{1-\gamma} g_{k}\right)=x^{1-\gamma} g_{i k}
\end{aligned}
$$

for some holomorphic functions $g_{k}^{\prime}, g_{i k}$ on $U(k=3,4 ; i=1,2)$.

$$
\begin{aligned}
\Delta= & \left|\begin{array}{cccc}
g_{1} & g_{2} & x g_{3} & x g_{4} \\
g_{1 x} & g_{2 x} & (1-\gamma) g_{3}+x g_{3 x} & (1-\gamma) g_{4}+x g_{4 x} \\
g_{1 y} & g_{2 y} & x g_{3 y} & x g_{4 y} \\
D_{2}\left(g_{1}\right) & D_{2}\left(g_{2}\right) & (1-\gamma)\left(-\gamma g_{3}+(y-1) g_{3 y}\right) & (1-\gamma)\left(-\gamma g_{4}+(y-1) g_{4 y}\right) \\
& & +x g_{3}^{\prime} & +x g_{4}^{\prime}
\end{array}\right| \\
& \times y^{2 \gamma^{\prime}} D^{-\gamma-\gamma^{\prime}+\alpha+\beta+3 / 2}
\end{aligned}
$$

is holomorphic in $U$, and the restriction $\left.\Delta\right|_{L_{x}}$ of $\Delta$ to $L_{x}$ is equall to

$$
\begin{aligned}
& \left|\begin{array}{cccc}
g_{1} & g_{2} & 0 & 0 \\
g_{1 x} & g_{2 x} & (1-\gamma) g_{3} & (1-\gamma) g_{4} \\
g_{1 y} & g_{2 y} & 0 & 0 \\
D_{2}\left(g_{1}\right) & D_{2}\left(g_{2}\right) & (1-\gamma)\left((y-1) g_{3 y}-\gamma g_{3}\right) & (1-\gamma)\left((y-1) g_{4 y}-\gamma g_{4}\right)
\end{array}\right| \\
& \times y^{2 \gamma^{\prime}}(y-1)^{2\left(-\gamma-\gamma^{\prime}+\alpha+\beta\right)+3} \\
& =-(1-\gamma)^{2}\left|\begin{array}{cc}
g_{1} & g_{2} \\
g_{1 y} & g_{2 y}
\end{array}\right|\left|\begin{array}{cc}
g_{3} & g_{4} \\
g_{3 y} & g_{4 y}
\end{array}\right| y^{2 \gamma^{\prime}}(y-1)^{2\left(-\gamma-\gamma^{\prime}+\alpha+\beta+2\right)}
\end{aligned}
$$

which is nonzero because both pairs $g_{1}, g_{2}$ and $g_{3}, g_{4}$ are linearly independent on $L_{x}$.

By the same reasoning, $\Delta_{k}^{i}$ are holomorphic on $U$ and $\Delta_{1}^{i}, \Delta_{3}^{i}$ vanish on $L_{x} \cap U$.

\subsubsection{Behavior along $L_{y}$}

For any point of $L_{y}-L_{x} \cup L_{\infty} \cup C$ there exists an open neibourhood $U$ so that $\Delta, \Delta_{k}^{i}$ are holomorphic on $U$ and that $\Delta_{2}^{i}, \Delta_{3}^{i}$ vanish on $L_{y} \cap U$. $\Delta$ is not zero along $L_{y}$.

\subsubsection{Behavior along $\boldsymbol{L}_{\infty}$}

For any point of $L_{\infty}-L_{x} \cup L_{y} \cup C$ there exists an open neighbourhood $U$ disjoint from $L_{x} \cup L_{y} \cup C$ and a basis $\xi^{\alpha} g_{1}, \xi^{\alpha} g_{2}, \xi^{\beta} g_{3}, \xi^{\beta} g_{4}$ of $\mathscr{F}$ satisfing 
$\left(\mathrm{EXP}_{\infty}\right) . \quad$ Then for $f_{k}=\xi^{\lambda} g_{k}(k=1,2,3,4 ; \lambda=\alpha, \beta)$

$$
\left(f_{k}\right)_{x}=\xi^{\lambda+1} f_{k 1},\left(f_{k}\right)_{y}=\xi^{\lambda+1} f_{k 2}, D_{2}\left(f_{k}\right)=\xi^{\lambda+1} f_{k 3}, Q_{i}\left(f_{k}\right)=\xi^{\lambda+1} f_{k i}^{\prime}
$$

for some holomorphic functions $f_{k j}, f_{k i}^{\prime}$. Since $x^{2 \gamma} y^{2 \gamma^{\prime}} D^{-\gamma-\gamma^{\prime}+\alpha+\beta+3 / 2}=$ $\xi^{-2 \alpha-2 \beta-3} u$ for some holomorphic function $u$ on $U, \Delta$ and $\Delta_{k}^{i}$ are holomorphic on $U$ and moreover the restrictions $\left.\Delta_{0}^{i}\right|_{L_{\infty}}$ of $\Delta_{0}^{i}$ to $L_{\infty}$ is zero $(i=1,2) . \Delta$ is not zero along $L_{\infty}$.

\subsubsection{Behavior along $C$}

For any point of $C-L_{x} \cup L_{y} \cup L_{\infty}$ there exist an open neighbourhood $U$ disjoint from $L_{x} \cup L_{y} \cup L_{\infty}$ and a basis $g_{1}, g_{2}, g_{3}, D^{\gamma+\gamma^{\prime}-\alpha-\beta-1 / 2} g_{4}$ of $\mathscr{F}$ satisfing $\left(\operatorname{EXP}_{C}\right)$. Put $f_{4}=D^{\gamma+\gamma^{\prime}-\alpha-\beta-1 / 2} g_{4}$ then $D_{2}\left(f_{4}\right) / D^{\gamma+\gamma^{\prime}-\alpha-\beta-3 / 2}$ and $Q_{i}\left(f_{4}\right) / D^{\gamma+\gamma^{\prime}-\alpha-\beta-3 / 2}$ are holomorphic on $U$. Hence $\Delta$ and $\Delta_{k}^{i}$ are all holomorphic on $U$.

\subsubsection{Behavior on $\mathbf{P}^{2}$}

We have proved that $\Delta$ and $\Delta_{k}^{i}$ are holomorphic on $\mathbf{P}^{2}$ except the six point $L_{x} \cap L_{y}, L_{x} \cap L_{\infty}, L_{y} \cap L_{\infty}, L_{x} \cap C, L_{y} \cap C, L_{\infty} \cap C$. By the Hartogs' extension theorem, $\Delta$ and $\Delta_{k}^{i}$ are holomorphic on $\mathbf{P}^{2}$. Since $\Delta_{k}^{i}$ vanish at $L_{x}$ or $L_{y}$ or $L_{\infty}, \Delta_{k}^{i}$ are identically zero.

Since $\Delta$ is not identically zero, $\Delta$ is a non-zero constant.

Thus by $\left(3.1 . \mathrm{EQ}_{i}\right), Q_{i}(f)=0$ and $\mathscr{F}$ coinsides with the solution space $\mathscr{F}_{4}$ of the Appell's differential equation $\left(F_{4}\right)$.

\section{Riemann's Problem of $\left(F_{4}\right)$ on $\mathrm{P}^{1} \times \mathrm{P}^{1}$}

Let

$$
\mathscr{F}=P\left\{\begin{array}{ccccc}
x=0 & y=0 & (x-y)^{2}-2(x+y)+1=0 & x=\infty & y=\infty \\
0 & 0 & 0 & \alpha & \alpha \\
0 & 0 & 0 & \beta & \beta \\
1-\gamma & 1-\gamma^{\prime} & 0 & 1+\alpha-\gamma^{\prime} & 1+\alpha-\gamma \\
1-\gamma & 1-\gamma^{\prime} & \gamma+\gamma^{\prime}-\alpha-\beta-\frac{1}{2} & 1+\beta-\gamma^{\prime} & 1+\beta-\gamma
\end{array}\right\}
$$

We replace the assumption $\left(\mathrm{EXP}_{\infty}\right)$ at the section 3 by the following $\left(\mathrm{EXP}_{x=\infty}\right)$ and $\left(\operatorname{EXP}_{y=\infty}\right)$ :

$\left(\operatorname{EXP}_{x=\infty}\right): \mathscr{F}$ has the exponent $\left(\alpha, \beta, 1+\alpha-\gamma^{\prime}, 1+\beta-\gamma^{\prime}\right)$ along $\{x=\infty\}$. 
This means, by definition, that at each point $P=(\infty, b)(b \neq 0, \infty)$ there exist an open neighbourhood $U$ and holomorphic functions $g_{1}, g_{2}, g_{3}, g_{4}$ on $U$ so that $x^{-\alpha} g_{1}, x^{-\beta} g_{2}, x^{-\left(1+\alpha-\gamma^{\prime}\right)} g_{3}, x^{-\left(1+\beta-\gamma^{\prime}\right)} g_{4}$ form a basis of $\mathscr{F}$ and that the restrictions of $g_{i}$ to $\{x=\infty\}$ are not identically zero.

$\left(\operatorname{EXP}_{y=\infty}\right): \mathscr{F}$ has the exponent $(\alpha, \beta, 1+\alpha-\gamma, 1+\beta-\gamma)$ along $\{y=\infty\}$.

Assume that none of $1-\gamma, 1-\gamma^{\prime}, \gamma+\gamma^{\prime}-\alpha-\beta-1 / 2$ is zero. Moreover we assume that $\alpha, \beta, 1+\alpha-\gamma^{\prime}, 1+\beta-\gamma^{\prime}$ are all distinct and also that $\alpha, \beta, 1+\alpha-\gamma, 1+\beta-\gamma$ are all distinct.

\section{1}

Fix a basis $f_{1}, f_{2}, f_{3}, f_{4}$ of $\mathscr{F}$. Let $f$ be any element of $\mathscr{F}$. Then we shall prove that $f$ belongs to $\mathscr{F}_{4}$ that is $Q_{1}(f)=Q_{2}(f)=0$, the notations are the same as in 3.1.

\subsection{Local behavior of $\Delta$ and $\Delta_{k}^{i}$ on $\mathrm{P}^{1} \times \mathrm{P}^{1}$}

\subsubsection{Behavior along $\{x=\infty\}$}

Assume that $g$ is of order $\lambda$ at $\{x=\infty\}$ that is $x^{\lambda} g$ is holomorphic at $\{x=\infty\}$ then $g_{y}, D_{2}(g), Q_{i}(g)$ are of order $\lambda$ and $g_{x}$ is of order $\lambda+1$. Hence $\Delta, \Delta_{k}^{i}$ $(k=0,2,3)$ are holomorphic along $\{x=\infty\}$. But $\Delta_{1}^{i}$ has at most a simple pole along $\{x=\infty\}$.

\subsubsection{Behavior along $\{\boldsymbol{y}=\infty\}$}

$\Delta, \Delta_{k}^{i}(k=0,1,3)$ are holomorphic along $\{y=\infty\}$ and $\Delta_{2}^{i}$ has at most simple pole along $\{y=\infty\}$.

\subsection{3}

$\Delta$ is holomorphic on $\mathbf{P}^{1} \times \mathbf{P}^{1}$ not identically zero. Hence $\Delta$ is a nonzero constant.

$\Delta_{0}^{i}$ is holomorphic on $\mathbf{P}^{1} \times \mathbf{P}^{1}$. Hence $\Delta_{0}^{i}=c^{i}$ for some constant $c^{i}$.

$\Delta_{3}^{i}$ is holomorphic on $\mathbf{P}^{1} \times \mathbf{P}^{1}$ and vanishes at $L_{x}$ and $L_{y}$. Hence $\Delta_{3}^{i}$ is zero.

$\Delta_{1}^{i}$ vanishes at $L_{x}$ and has at most simple pole along $\{x=\infty\}$ and holomorphic elsewhere. Hence $\Delta_{1}^{i}=c_{1}^{i} x$ for some constant $c_{1}^{i}$. $\Delta_{2}^{i}=c_{2}^{i} y$ for some constant $c_{2}^{i}$.

4.3

The equation $\left(3.1 . \mathrm{EQ}_{i}\right)$ now takes the form

$$
Q_{i}(f)-c_{1}^{i} x f_{x}+c_{2}^{i} y f_{y}+c^{i} f=0 \quad i=1,2 .
$$


We will show that $c_{k}^{i}$ and $c^{i}$ are all zero. Let $\xi=\frac{1}{x}$ and $f=\xi^{i}\left(\varphi_{1}(y)\right.$ $\left.+\xi \varphi_{1}(y)+\xi^{2} \varphi_{2}(y)+\cdots\right)$ be a solution of (4.3.EQ $\left.\mathrm{EQ}_{i}\right)\left(\lambda=\alpha, \beta, 1+\alpha-\gamma^{\prime}\right.$, $\left.1+\beta-\gamma^{\prime}\right)$. Taking the cocfficients of $\xi^{\lambda}$ in (4.3.EQ $\left.\mathrm{E}_{i}\right)$, we obtain two differential equations $E_{i}\left(\varphi_{0}\right)$.

$$
\begin{aligned}
E_{1}\left(\varphi_{0}\right):-y^{2} \varphi_{0}^{\prime \prime}+(2 \lambda-\alpha-\beta-1) y \varphi_{0}^{\prime}-(\lambda-\alpha)(\lambda-\beta) \varphi_{0} \\
-c_{1}^{1} \lambda \varphi_{0}+c_{2}^{1} y \varphi_{0}^{\prime}+c^{1} \varphi_{0}=0 \\
E_{2}\left(\varphi_{0}\right): y(1-y) \varphi_{0}^{\prime \prime}+\left(\gamma^{\prime}+(2 \lambda-\alpha-\beta-1) y\right) \varphi_{0}^{\prime}-(\lambda-\alpha)(\lambda-\beta) \varphi_{0} \\
-c_{1}^{2} \lambda \varphi_{0}+c_{2}^{2} y \varphi_{0}^{\prime}+c^{2} \varphi_{0}=0
\end{aligned}
$$

Eliminating $\varphi_{0}^{\prime \prime}, \varphi_{0}^{\prime}$ from $E_{1}\left(\varphi_{0}\right), E_{2}\left(\varphi_{0}\right)$ we obtain an equation $E(\lambda)=0$, where $E(\lambda)=\lambda^{4}+\cdots$ is a polynomial of $\lambda, y, \alpha, \beta, \gamma, \gamma^{\prime}, c_{j}^{i}, c^{i}$. The roots of $E(\lambda)=0$ must be $\alpha, \beta, 1+\alpha-\gamma^{\prime}, 1+\beta-\gamma^{\prime}$. This happens only when $c_{1}^{1}=c_{2}^{1}=c^{1}=0$. By the same consideration at $y=\infty$, we also have $c_{1}^{2}=c_{2}^{2}=c^{2}=0$. to $\mathscr{F}_{4}$.

This proves that any $f$ in $\mathscr{F}$ satisfies $Q_{i}(f)=0 i=1,2$, that is, $f$ belongs

\section{References}

[1] P. Appell, J. Kampé de Fériet, Fonctions Hypergéométriques et Hypersphériques, Gauthier Villars, Paris, 1926.

[2] M. Kato, A Pfaffian system of Appell's $F_{4}$, Bulletin of College of Education Univ. of the Ryukyus, No. 33 (1988) 331-334.

[3] T. Terada, Probleme de Riemann et fonctions automorphes provenant des fonctions hypergéométriques de plusieurs variables, J. Math. Kyoto Univ., 13 (1973), 557-578.

Mitsuo KATO.

Department of mathematics

College of Education

University of the Ryukyus

Nishihara-cho, Okinawa 903-01, JAPAN 